Ludwig M. Eichinger (Mannheim)

\title{
Sprachliche Vielfalt und gesellschaftliche Diversität: Das Deutsch der Migranten
}

\section{Die sprachliche Vielfalt moderner europäischer Gesellschaften}

Die 48. Jahrestagung des Instituts für Deutsche Sprache beschäftigte sich mit einem sprachlichen Thema, das auf ein hohes gesellschaftliches Interesse trifft. Seit einiger Zeit ist klar geworden, dass die Arbeitsmigration, die seit etwa 50 Jahren den Aufbau der Gesellschaft in Deutschland beeinflusst, nicht etwas Vorübergehendes ist, und daher auch sprachliche Verhältnisse schafft und sprachliche (und darüber hinaus gehende) Entwicklungen auslöst, die ihrerseits nicht irgendwie etwas Vorläufiges und Vorübergehendes darstellen.

Vor allem die städtischen Gesellschaften der Bundesrepublik Deutschland - über die anderen deutschsprachigen Staaten soll hier nichts gesagt werden - sind durch einen hohen Grad an Multidimensionalität, die Existenz und Konkurrenz einer Vielzahl von Interaktionsgemeinschaften geprägt. Diese Gemeinschaften sind zweifellos Zentren einer verdichteten Interaktion, zudem sind sie daher systemisch darauf angelegt, diese Verdichtung auch symbolisch zu kennzeichnen. Dennoch sind diese Einheiten keine abgeschlossenen Diskurskreise. In modernen westeuropäischen Gesellschaften geht das weit über den Tatbestand hinaus, dass es Spezialisten für die Kommunikation zwischen den verschiedenen Gruppen bzw. zur Mehrheitsgemeinschaft hin gäbe. Vielmehr interagieren auf der Ebene der Individuen Partner mit unterschiedlichen aber hoch diversifizierten Profilen miteinander. Wie weit das einen Zustand der vielfachen und vielfältigen Fusion kommunikativer Optionen hervorgebracht hat, der es kaum mehr erlaubt, in einer einfachen Weise von gesellschaftlicher Mehrsprachigkeit zu sprechen, wird unter dem Leitmodell eines qualitativen Sprungs in der Dimensionalität (,superdiversity“) diskutiert. ${ }^{1}$ Darin mögen sich die verschiedenen städtischen Gesellschaften Europas unterscheiden, der Tatbestand einer grundlegenden Überlagerung der sprachlichen Verhältnisse in unseren Gesellschaften ist unstrittig. Für die bundesdeutschen städtischen Interaktionsräume hat sich der sprachliche Alltag in einer Weise verändert, der mit der Beschreibung mehr oder minder isolierter "anderssprachiger" Gruppen nicht angemessen beschrieben wäre. Vielmehr stellen nach dieser

Vgl. dazu Rampton (2006, S. 11 ff.); Vertovec (2007). 
jahrzehntelangen Entwicklung seit dem Beginn eines großräumigeren $\mathrm{Zu}$ zugs von Personengruppen, die aus anderssprachigen Räumen stammen, heutzutage sprachliche Formen und Ausdrucksweisen, deren Ursprung auf entsprechende Sprachkontaktphänomene zurückzuführen ist, einfach einen Teil des alltäglichen sprachlichen Interaktionsgefüges in diesen gesellschaftlichen Formationen dar. Beginn und mengenmäßiger Kern dieses Zuzugs war ein Typ von Arbeitsmigration, der erklärt, warum sich solche sprachlichen Erscheinungen vor allem in Städten finden und in der generationellen Folge zudem bevorzugt bei Gruppen von Sprecherinnen und Sprechern, die eher jünger sind und einem informellen Lebensstil zuneigen. Bemerkenswert ist auch, dass diese sprachlichen Formen und Routinen unter spezifischen Bedingungen allmählich so weit in unser sozialpsychologisches Orientierungssystem eingebaut sind, dass sie nicht als ethnolektale Sprechweisen auf die „Ursprungsgruppe“ mit entsprechendem fremdsprachlichem Hintergrund begrenzt bleiben, sondern im Sinne gesellschaftlicher Symbolisierungsroutinen genutzt werden - wenn auch in begrenztem Ausmaß. Aus verschiedenen Gründen ist es nicht besonders überraschend, dass dieser Effekt insbesondere in gesellschaftlichen Kontexten aufscheint, in denen jugendliche Identitäten sich über Bilder innerstädtisch-progressiver Lebensstile konstituieren, Lebensstile, deren Imagologie entlang leitender jugendkultureller Vorstellungen von großstädtischer Härte geprägt ist. ${ }^{2}$

\section{Migration und ihre Folgen in Deutschland}

Diese sprachlich-kulturellen Veränderungen sind nicht zuletzt vor dem Hintergrund zu sehen, dass ihre Entstehungsgrundlage - der Sprachkontakt im Kontext und im Gefolge von (Arbeits-)Migration - quantitativ ganz erhebliche Ausmaße angenommen hat. Dass diese quantitative Seite längere Zeit nicht so sehr in den Fokus geriet, hat nicht zuletzt damit zu tun, dass die Zahlenverhältnisse im Hinblick auf die „Personen mit Migrationshintergrund" in der bundesdeutschen Bevölkerung erst seit den MikrozensusErhebungen des letzten Jahrzehnts einigermaßen konkret fassbar und auch in ihrer regionalen und sozialen Verteilung erfassbar sind. ${ }^{3}$

Die verschiedenen Stadien - vielleicht auch Stadien der Wahrnehmung - dieser Entwicklung spiegeln sich auch in der Art der wissenschaftlichen Fragestellungen, die von ihr ausgelöst werden. Das ergibt über die Zeit gesehen eine ganz erhebliche Bandbreite an Fragestellungen, die in diesem Kontext wissenschaftliche - soziolinguistische - Themen darstellen. War das Bild dieser Migration zu Beginn in der Öffentlichkeit vom Bild der auf Zeit angeworbenen Gastarbeiter geprägt, so passen dazu auch die ersten Unter-

Dazu und zu den Abstufungen des Ethnolektalen siehe Auer (2003).

Zu den neuesten Daten siehe Statistisches Bundesamt (Hg.) (2011). 
suchungen, die unter dem Titel laufen: „Das Pidgin-Deutsch der Gast$\operatorname{arbeiter~}^{\text {"4 }}$ (das damals an spanischen und italienischen Gastarbeitern untersucht wurde).

Als die gesellschaftliche Frage des zumindest längeren Aufenthalts der Zugewanderten allmählich virulenter wurde, begannen sich auch die wissenschaftlichen Untersuchungen auf Fragen der Verhältnisse bei ersten und zweiten Generationen bzw. im familiären Kontext auszuweiten. ${ }^{5}$ Mit der politischen Wende der 1990er Jahre, als dann neben der Frage der Aussiedler auch die Frage von Asylsuchenden für die bundesdeutsche Gesellschaft relevant wurde - nicht zuletzt aufgrund der Kriegsfolgen im ehemaligen Jugoslawien -, kommen zudem die immer komplexer werdenden sprachlichen Verhältnisse in den Fokus der Untersuchungen. In der Folge dieser Diskussion kam es zu Klärungsprozessen im Hinblick auf den Status der Bundesrepublik Deutschland als eines „Einwanderungslandes“,, 6 die dann auch zu einem Zuwanderungsgesetz mit seinen Folgeregelungen führten. ${ }^{7}$ Das scheint die Stufe zu sein, zu der die Wissenschaft von der Frage bewegt wird, wie eine den demokratischen Verhältnissen angemessene sprachliche Integration aussehen sollte. ${ }^{8}$

Wenn man die Abfolge dieser Etappen der Diskussion betrachtet, so ist es ein weiter Weg von den Anfängen der „Gastarbeiterlinguistik“ zu den Konzepten und Konstruktionen, die sich unter dem Titel dieser Tagung „Das Deutsch der Migranten“ finden.

\section{Gibt es das Deutsch der Migranten?}

Kurze Titel verkürzen: Nicht dass der Titel der Tagung, die in diesem Band dokumentiert ist, in seiner Kürze nicht verschiedene Interpretationen zuließe. Sie lassen sich als Kondensationen aus Texten unter Weglassen von Einzel-

$4 \quad$ So der Titel des einflussreichen Beitrags Clyne (1968), der den Anstoß zu den Untersuchungen dieser frühen Phase gab; vgl. Riehl (2004, S. 107 ff.). Die bedeutendste Studie in diesem Kontext war das Heidelberger Forschungsprojekt (Klein 1975).

5 Vgl. dazu z.B. Cindark (2011).

6 Siehe die entsprechenden Karten in Dorling/Newman/Barford (2010) zur Einwanderung und zum „Nettogewinn“ an Bevölkerung durch Migration.

Vgl. neben dem Zuwanderungsgesetz (2004) z.B. die in Goethe-Institut (2012) gegebenen Informationen zu den praktischen Folgen.

8 Dass es für solche Überlegungen eher hinderlich ist, dass es keine statistisch verlässliche Grundlage zu den in Deutschland gesprochenen Sprachen gibt, kann man zu recht beklagen, vgl.: „In der amtlichen Statistik gibt es keine Datenquelle, die über Sprachkenntnisse der ausländischen Bevölkerung oder der Personen mit Migrationshintergrund in Deutschland Auskunft geben könnte. So enthält beispielsweise der Mikrozensus des Statistischen Bundesamtes keine Fragen und Angaben zu Sprachkenntnissen. Insofern ist die Datenlage im Bereich der sprachlichen Integration im Vergleich zu anderen Integrationsbereichen relativ schlecht" (Haug 2008, S. 11); anders ist das z.B. in den USA (siehe US Census 2010), wo nach anderen Sprachen, die in der Familie gesprochen werden, gefragt wird. 
heiten verstehen, und können so durchaus die Titel von unterschiedlichen Geschichten sein. Wenn hier einfach ohne weitere Attribution von Deutsch, von der deutschen Sprache also, die Rede ist, spricht das zunächst einmal davon, dass die gemeinten sprachlichen Befunde nicht mehr als irgendetwas Äußeres, sondern als ein funktional und sozial lesbarer Bestandteil der „normalen“ Kommunikation im deutschen Sprachraum gesehen werden. Daraus kann man allerdings noch keine konkreteren Schlüsse darauf ziehen, wie man die in diesem Kontext beobachteten Sprachformen, Ethnolekte, Entwicklungen und Trends im Varietätenkontext des Deutschen zu positionieren hätte, ${ }^{9} \mathrm{ob}$ und wie weit man überhaupt von einer Varietät in einem irgendwie abgeschlossenen Sinne sprechen will. Auf der anderen Seite ist auch der Gebrauch des Wortes Migrant - seit es denn gängig ist ${ }^{10}$ nicht eindeutig. Das prototypische Konzept, das hinter den Verwendungen dieses Wortes sichtbar wird, ist nicht so einheitlich - nicht alle Personen mit Migrationshintergrund, wie es in der Terminologie der Statistiker des Mikrozensus heißt, gelten in gleicher prototypischer Weise als Migranten bzw. auch als typische Repräsentanten der entsprechenden Sprachformen. ${ }^{11}$ Es sind die großen Gruppen, die bzw. deren Eltern und Großeltern in die Bundesrepublik Deutschland gekommen sind und bei denen die Vorstellung einer größeren sprachlichen und kulturellen Differenz zum „autochthonen" Deutsch herrscht, die das allgemeine Bild und die vorherrschenden gesellschaftlichen Vorstellungen davon prägen. ${ }^{12}$

Prominent ist etwa die Gruppe der Personen mit Bezug auf die Türkei bzw. das Türkische, deutlich weniger gilt eine solche Zuordnung für eine - zweifellos ebenfalls durch Migration nach Deutschland gekommene - andere Gruppe, von der auf der Tagung die Rede war, die Russlanddeutschen, und der Beitrag zu den nach Israel ausgewanderten Deutschen, der sich ebenfalls in diesem Band findet, zeigt, dass Migration auch noch ganz andere Gesichter und Erscheinungsformen hat. ${ }^{13}$ All diese Zuordnungen stellen natürlich auch den Rahmen dafür dar, wie in öffentlichen und nicht zuletzt schulischen Kontexten mit dem Deutsch der Migranten umzugehen ist.

Es ist - was nach diesen Ausführungen nur logisch ist - das erste Mal, dass es eine Jahrestagung unter dieser Themenformulierung gibt, Thema

\footnotetext{
$9 \quad$ Vgl. dazu jetzt den weit gehenden Vorschlag von Wiese (2012) bzw. die Beiträge von Auer und Wiese in diesem Band.

10 Seit etwa Mitte der 1990er Jahre.

11 In gewissem Umfang spiegeln sich diese Einschätzungen in den Meinungen zu fremdsprachigen Akzenten, die in Gärtig/Plewnia/Rothe (2010) dokumentiert sind.

12 Nach dem Mikrozensus von 2010 sind die Personen mit türkischem Hintergrund bei weitem die größte Gruppe (ca. 2,5 Mio.), gefolgt von Personen mit polnischem Migrationshintergrund (ca. 1,3 Mio.); alle weiteren Gruppen sind deutlich kleiner. Allerdings erreicht die Gruppe mit Bezug auf die alte Sowjetunion ungefähr die Größe der türkischen Gruppe und die des ehemaligen Jugoslawien die der polnischen; siehe Statistisches Bundesamt (Hg.) (2011).

13 Vgl. die Beiträge von Berend und Betten in diesem Band.
} 
war die zugrunde liegende Frage aber zumindest schon einmal, im Jahr 1981, eine Gelegenheit, die den Beginn einer jahrzehntelang anhaltenden Beschäftigung mit diesem Thema am IDS darstellte. Das Thema hieß damals „Mehrsprachigkeit in der Stadtregion“, ${ }^{14}$ Werner Kallmeyer und Inken Keim stellten bei dieser Gelegenheit das auf die Mannheimer Verhältnisse bezogene Projekt „Kommunikation in der Stadt“ vor, das 1994 und 1995 seine Ergebnisse in vier dicken Bänden zur Stadtsprache darstellte. ${ }^{15}$ Inken Keim führte die dort begonnenen Untersuchungen zu den kommunikativen Praktiken auf das Türkische bezogener Sprechergruppen über die Jahrzehnte hin fort, ${ }^{16}$ und war auch in diesem Jahr mit einem Vortrag vertreten, in dem sie gemeinsam mit einem ihrer alten Projektmitarbeiter verschiedene Sprechertypen skizziert, die sich in diesem Kontaktfeld herausgebildet haben. ${ }^{17} \mathrm{Ge}$ sprochen hat von den diesjährigen Referenten damals auch schon Peter Auer (Auer 1982), und bei der Publikation dabei war auch schon Peter Schlobinski, dem nun im Jahr 2012 der Konrad-Duden-Preis der Stadt Mannheim verliehen wurde. ${ }^{18}$

Erkennbar ist bei der Lektüre der damaligen Beiträge, dass es damals mehr um die Beziehungen von zwei getrennten Sprachen ging, als um die Frage der Integration der vorhandenen sprachlichen Optionen in ein Spektrum kommunikativer Möglichkeiten. So wird der Befund dort - im Text auf dem Rücken des Tagungsbandes (Bausch (Hg.) 1982) - folgendermaßen geschildert:

Der Kontakt zwischen Deutschen und Ausländern führte zur Übernahme von Elementen der Fremdsprache in die deutsche Sprache. Er führte aber auch dazu, dass sich unter den Ausländern Sprachformen der Muttersprache entwickeln, die durchmischt sind mit den Elementen des Deutschen. Und schließlich entwickeln sich im Kontakt zwischen Ausländern mit verschiedenen Muttersprachen neue Formen des Deutschen.

Heute geht es uns nach den zwischenzeitlichen Erfahrungen um Anderes, um die Frage der Integration ursprünglich kontaktinduzierter sprachlicher Formen und Züge in die gesellschaftliche Interaktion, und es geht daher nicht zuletzt um die praktischen Folgen, ${ }^{19}$ die in einer modernen multidimensionalen Gesellschaft ${ }^{20}$ daraus abzuleiten sind.

\footnotetext{
Siehe Bausch (Hg.) (1982).

Siehe Debus/Kallmeyer/Stickel (Hg.) (1994-1995).

Siehe Keim (2008).

Siehe Cindark (in diesem Band).

Siehe Schlobinski (2012); Dittmar/Schlieben-Lange/Schlobinski (1982).

19 Vgl. dazu etwa das in Stanat/Rauch/Segeritz (2010) gegebene Resümee zu den entsprechenden PISA-Befunden; siehe auch die im Hinblick auf Mehrsprachigkeitsgewinne eher skeptischen Ausführungen in Esser (2006).

20 Wie weit hier die sprachlichen Kontakt- und Amalgamierungsprozesse gehen, wie weit man sprachliche Differenzen als Epiphänomene einer Konstruktion von Identität verstehen kann (vgl. dazu neben Rampton in diesem Band etwa Blommaert/Rampton 2011) oder doch auch
} 


\section{Mannheim als Exempel - und wofür?}

Die Stadtsprache Mannheims zu untersuchen, hatte das IDS sich im Jahr 1981 vorgenommen ${ }^{21}$ als unübersehbarer Teil dieses Untersuchungsobjekts blieb das Sprechen und die Kommunikation der Migranten seither ein Thema der Forschungen im Institut. ${ }^{22}$

Mannheim ist nach wie vor ein guter Ort für solche Untersuchungen, mit etwa 38\% Wohnbevölkerung mit Migrationshintergrund - wie Stuttgart - nimmt es nach Frankfurt, das von den deutschen Städten mit etwa 43\% in dieser Kategorie die höchste Quote hat, eine der ersten Stellen ein ${ }^{23}$ (in Berlin sind es zum Vergleich etwa 24,3\%), von den Flächenländern hat zudem Baden-Württemberg im Bundesdurchschnitt die höchste Quote an Personen mit Migrationshintergrund (mit 26\%; Bundesdurchschnitt 19\%). ${ }^{24}$ Man sieht hier andeutungsweise schon, dass auch innerhalb der bundesdeutschen Verhältnisse Differenzen vorhanden sind, von denen die Deutungen nicht unbeeinflusst bleiben können - und bei deren Größenordnung ${ }^{25}$ man sich auch einmal von sprachwissenschaftlicher Seite quantitative Untersuchungen zu den sprachlichen Verhältnissen wünschen würde.

\section{Schluss}

An den Themen, die in den Beiträgen dieses Bandes behandelt werden, kann man sehen, dass das Bewusstsein um die Notwendigkeit einer angemessenen öffentlichen - auch praktischen - Haltung zu diesen doch weiträumigen Veränderungen in der öffentlichen Diskussion angekommen ist. Wenn wir uns als Sprachwissenschaftler von der sprachlichen Seite an die Sache annähern, ist der zentrale Ort dieser Fragen daran erkennbar, dass ganz unterschiedliche Konzeptualisierungen des zu untersuchenden Gegen-

als Phänomene erfahrener Differenz, hängt sicher unter anderem vom jeweiligen gesellschaftlichen Umfeld und seinen Traditionen ab (vgl. die Antworten zur Wahrnehmung „Anderssprachiger in Eichinger et al. 2009 und Gärtig/Plewnia/Rothe 2010), und wird sicher auch sprachwissenschaftlich weiter zu diskutieren sein (siehe z.B. die Beiträge in Eichinger/ Plewnia/Steinle (Hg.) 2011; v.a. Stevenson 2011)

21 Siehe Kallmeyer/Keim/Nikitopoulos (1982).

22 Ein in diesem Rahmen durchgehendes Forschungsthema ist dokumentiert in Keim (2008), aber auch bei Cindark (2011).

23 In Anbetracht dessen mag der folgende Befund die Frage von Integriertheit und Integration noch einmal von einer anderen Seite zu beleuchten: „Bemerkenswert ist aber auch, dass einige Städte mit hohen Anteilen an Migrantinnen und Migranten an der Bevölkerung, unter ihnen Mannheim, Heilbronn, Ingolstadt, Hagen und Pforzheim, kein Ratsmitglied mit Migrationshintergrund aufweisen" (Schönwälder/Sinanoglu/Volkert 2011, S. 12).

24 Daten nach Statistisches Bundesamt (Hg.) (2011) und Stadt Mannheim (2012).

25 In Mannheim handelt es sich immerhin um ca. 123.000 Personen mit Migrationshintergrund, davon gehören 28.300 (23\%) zu der größten Gruppe, der mit türkischem Migrationshintergrund. In Berlin lauten die entsprechenden Zahlen 835.000 und 176.000 (21\%). 
standsbereichs vorgeschlagen und diskutiert werden. Die eine Seite dabei stellt die Frage dar, welchen Wert man der zu beobachtenden sprachlichen Variation vor dem Hintergrund gängiger Beschreibungen des Deutschen beimisst. Strittig und in gewisser Weise entscheidend ist dabei die Frage, inwieweit hierbei Hinweise auf gesellschaftliche Mehrsprachigkeit überhaupt nützlich sind, um das individuelle und das Gruppen-Sprachverhalten in den beobachteten gesellschaftlichen Gruppen zu erklären. Das hat dann natürlich auch Folgen für die Frage, wie man die praktischen Seiten dieser Entwicklungen - etwa in der schulischen Praxis - angeht. Dass dazu der Blick über die Bundesrepublik Deutschland hinaus ebenso wichtig und erkenntnisträchtig ist wie der Blick über die engeren Fachgrenzen hinaus, hat auch die hier dokumentierte Tagung ${ }^{26}$ wieder gezeigt. ${ }^{27}$

\section{Literatur}

Auer, Peter (1982): Transferierte Rituale in bilingualen Interaktionen italienischer Migrantenkinder. In: Bausch (Hg.), S. 194-224.

Auer, Peter (2003): ,Türkenslang‘: Ein jugendsprachlicher Ethnolekt des Deutschen und seine Transformationen. In: Häcki Buhofer, Annelies (Hg.): Spracherwerb und Lebensalter. (= Basler Studien zur deutschen Sprache und Literatur 83). Tübingen/Basel, S. 255-264.

Auer, Peter (in diesem Band): Ethnische Marker im Deutschen zwischen Varietät und Stil.

Bausch, Karl-Heinz (Hg.) (1982): Mehrsprachigkeit in der Stadtregion. (= Jahrbuch 1981 des Instituts für deutsche Sprache). Düsseldorf.

Berend, Nina (in diesem Band): Varietätenwandel im Kontakt. Die Entwicklung des Sprachgebrauchs deutschsprachiger Minderheiten im bundesdeutschen Kontext.

Betten, Anne (in diesem Band): Sprachbiographien deutscher Emigranten. Die „Jeckes“ in Israel zwischen Verlust und Rekonstruktion ihrer kulturellen Identität.

Blommaert, Jan/Rampton, Ben (2011): Language and superdiversity. In: Diversities 13, 2, S. 1-21.

Cindark, Ibrahim (2011): Migration, Sprache und Rassismus. Der kommunikative Sozialstil der Mannheimer „Unmündigen“ als Fallstudie für die „emanzipatorischen Migranten“. (= Studien zur Deutschen Sprache 51). Tübingen.

26 Zum Vorbereitungsteam der Tagung gehörten Angelika Redder, Rosemarie Tracy, Inken Keim, Nina Berend und Arnulf Deppermann; ihnen sei auch hier noch einmal gedankt wie auch allen Referentinnen und Referenten für Ihre Beiträge.

27 Kurz vor der Tagung war die Nachricht von dem unzeitigen Tod von Frau Dr. Susanne Anschütz bekannt geworden. Die Bedeutung von Frau Anschütz für die sprachwissenschaftliche Forschung ist mit ihrer Tätigkeit als Referentin in der DFG für dieses Fachgebiet zwar bezeichnet, aber in seiner Bedeutung für die Sprachwissenschaft und viele Sprachwissenschaftler/innen nicht hinreichend beschrieben. Ihrer wurde bei der Eröffnung der Tagung gedacht und das soll auch an diesem Orte nicht vergessen sein. 
Cindark, Ibrahim (in diesem Band): Deutsch-türkisches Code-Switching und CodeMixing in einer Gruppe von akademischen Migranten der zweiten Generation

Clyne, Michael (1968): Zum Pidgin-Deutsch der Gastarbeiter. In: Zeitschrift für Mundartforschung 35, S. 130-139.

Debus, Friedhelm/Kallmeyer, Werner/Stickel, Gerhard (Hg.) (1994-1995): Kommunikation in der Stadt. 4 Bde. (= Schriften des Instituts für Deutsche Sprache 4.1-4.4). Berlin/New York.

Dittmar, Norbert/Schlieben-Lange, Brigitte/Schlobinski, Peter (1982): Teilkommentierte Bibliographie zur Soziolinguistik von Stadtsprachen. In: Bausch (Hg.), S. 391-423.

Dorling, Daniel/Newman, Mark/Barford, Anna (2010): Atlas der wirklichen Welt. So haben Sie die Erde noch nie gesehen. Darmstadt.

Eichinger, Ludwig M./Plewnia, Albrecht/Steinle, Melanie (Hg.) (2011): Sprache und Integration. Über Mehrsprachigkeit und Migration. (= Studien zur Deutschen Sprache 57). Tübingen.

Eichinger, Ludwig M. et al. (2009): Aktuelle Spracheinstellungen in Deutschland. Erste Ergebnisse einer bundesweiten Repräsentativumfrage. Mannheim.

Eichinger, Ludwig M. et al. (Hg.) (2012): Sprache und Einstellungen. Spracheinstellungen aus sprachwissenschaftlicher und sozialpsychologischer Perspektive. Mit einer Sprachstandserhebung zum Deutschen von Gerhard Stickel. (= Studien zur Deutschen Sprache 61). Tübingen.

Esser, Hartmut (2006): Sprache und Integration. Die sozialen Bedingungen und Folgen des Spracherwerbs von Migranten. Frankfurt a.M./New York.

Gärtig, Anne-Kathrin/Plewnia, Albrecht/Rothe, Astrid (2010): Wie Menschen in Deutschland über Sprache denken. Ergebnisse einer bundesweiten Repräsentativerhebung zu aktuellen Spracheinstellungen. (= amades 40). Mannheim.

Goethe-Institut (Hg.) (2012): Sprache und Integration. München. Internet: www.goethe. de/lhr/prj/daz/pro/Goethe-Institut_Sprache_und_Integration_2012.pdf (Stand: 05.10.2012).

Haug, Sonja (2008): Sprachliche Integration von Migranten in Deutschland. (= Bundesamt für Migration und Flüchtlinge: Working paper der Forschungsgruppe des Bundesamtes 14; Integrationsreport 2). Nürnberg.

Kallmeyer, Werner/Keim, Inken/Nikitopoulos, Pantelis (1982): Zum Projekt „Kommunikation in der Stadt“. In: Bausch (Hg.), S. 345-391.

Keim, Inken (2008): Die „türkischen Powergirls“. Lebenswelt und kommunikativer Stil einer Migrantinnengruppe in Mannheim. 2., durchges. Aufl. (= Studien zur Deutschen Sprache 39). Tübingen.

Klein, Wolfgang (1975): Sprache und Kommunikation ausländischer Arbeiter. Heidelberger Forschungsprojekt „Pidgin-Deutsch“. (= Monographien Linguistik und Kommunikationswissenschaft 20). Kronberg.

Rampton, Ben (2006): Language in late modernity: interaction in an urban school. (= Studies in Interactional Sociolinguistics 22). Cambridge.

Rampton, Ben (in diesem Band): From 'Youth Language' to contemporary urban vernaculars. 
Riehl, Claudia Maria (2004): Sprachkontaktforschung. Eine Einführung. Tübingen.

Schlobinski, Peter (2012): Sprache und Kommunikation im digitalen Zeitalter. Rede anlässlich der Verleihung des Konrad-Duden-Preises der Stadt Mannheim am 14. März 2012. Laudatio von Ludwig M. Eichinger. (= Dudenbeiträge zu Fragen der Rechtschreibung, der Grammatik und des Stils 61). Mannheim/Zürich.

Schönwälder, Karen/Sinanoglu, Cihan/Volkert, Daniel (2011): Vielfalt sucht Rat. Ratsmitglieder mit Migrationshintergrund in deutschen Großstädten. Eine Studie des Max-Planck-Instituts zur Erforschung multireligiöser und multiethnischer Gesellschaften. (= Schriften zur Demokratie 27). Berlin.

Stadt Mannheim, Kommunale Statistikstelle (2012): Bevölkerung mit Migrationshintergrund (Wohnberechtigte) zum 31.12.2011. Internet: www.mannheim.de/stadt-gestalten /einwohner-migrationshintergrund (Stand: 05.10.2012).

Stanat, Petra/Rauch, Dominique/Segeritz, Michael (2010): Schülerinnen und Schüler mit Migrationshintergrund. In: Klieme, Eckhard et al. (Hg.): PISA 2009. Bilanz nach einem Jahrzehnt. Münster u.a., S. 200-230.

Statistisches Bundesamt (Hg.) (2011): Bevölkerung und Erwerbstätigkeit: Bevölkerung mit Migrationshintergrund. Ergebnisse des Mikrozensus 2010. Wiesbaden: Statistisches Bundesamt. Internet: www.destatis.de/DE/Publikationen/Thematisch/Bevoelkerung /MigrationIntegration/Migrationshintergrund2010220107004.pdf?_blob=publicationFile. (Stand: 05.10.2012).

US Census 2010. Internet: http://2010.census.gov/2010census/data/ (Stand: 05.10.2012).

Vertovec, Steven (2007): Super-diversity and its implications. In: Ethnic and Racial Studies 30, S. 1024-1054.

Wiese, Heike (in diesem Band): Das Potenzial multiethnischer Sprechergemeinschaften.

Wiese, Heike (2012): Kiezdeutsch. Ein neuer Dialekt entsteht. München.

Zuwanderungsgesetz (2004): Gesetz zur Steuerung und Begrenzung der Zuwanderung und zur Regelung des Aufenthalts und der Integration von Unionsbürgern und Ausländern (Zuwanderungsgesetz). Vom 30. Juli 2004. Berlin. 
